\title{
Inverse Nodal Problem for a Class of Nonlocal Sturm-Liouville Operator
}

\author{
Chuan-Fu Yang \\ Department of Applied Mathematics, \\ Nanjing University of Science and Technology \\ Nanjing 210094, Jiangsu, People's Republic of China \\ E-mail: chuanfuyang@tom.com
}

Received June 7, 2009; revised April 1, 2010; published online July 15, 2010

\begin{abstract}
Inverse nodal problem consists in constructing operators from the given nodes (zeros) of their eigenfunctions. In this work, the Sturm-Liouville problem with one classical boundary condition and another nonlocal integral boundary condition is considered. We prove that a dense subset of nodal points uniquely determine the boundary condition parameter and the potential function of the Sturm-Liouville equation. We also provide a constructive procedure for the solution of the inverse nodal problem.
\end{abstract}

Keywords: Sturm-Liouville problem, nonlocal integral condition, inverse nodal problem, potential function, reconstruction formula.

AMS Subject Classification: 34B05.

\section{Introduction}

Boundary problems with nonlocal conditions are a part of fast developing differential equations theory. Problems of this type arise in various fields of physics, biology, biotechnology and etc. Sturm-Liouville problems with integral conditions constitute a very interesting class of problems since they include as special cases two-, three- and multi-point boundary conditions. Nonlocal conditions come up when value of the function on the boundary is connected to values inside the domain. Theoretical investigation of problems with various types of nonlocal boundary conditions is actual problem and recently it is paid much attention for them in the literature. Originators of such problems were Samarskii and Bitsadze [2].

Quite a new area, related to problems of this type, deals with investigation of the spectrum of differential equations with nonlocal conditions. Eigenvalue problems with nonlocal conditions are closely linked to boundary problems for diffrential equations with nonlocal conditions $[6,11,13,14]$. In the papers $[9,24,30,25,26]$ the similar problems are investigated for the operators with 
nonlocal condition of Bitsadze-Samarskii or integral type. Albeverio, Hryniv and Nizhnik [1] solve the inverse spectral problem for a class of Sturm-Liouville operators with singular non-local potentials and non-local boundary conditions.

Inverse spectral problems consist in recovering operators from their spectral characteristics. Such problems play an important role in mathematics and have many applications in natural sciences (see, for example, [19, 21, 23, 32, 33]). In 1988, the inverse nodal problem was posed and solved for Sturm-Liouville problems by J. R. McLaughlin [22], who showed that knowledge of a dense subset of nodal points of the eigenfunctions is sufficient to determine the potential function of the Sturm-Liouville problem up to a constant. Some numerical schemes were provided by O. H. Hald and J. R. McLaughlin [12] for the reconstruction of the potential. This is the so-called inverse nodal problem. From the physical point of view this corresponds to finding, e.g., the density of a string or a beam from the zero-amplitude positions of their eigenvibrations. Recently, some authors have reconstructed the potential function for generalizations of the Sturm-Liouville problem from the nodal points (for example, refer to $[3,5,7,8,10,12,15,16,17,18,22,27,28,29,31,34])$. When it comes to Sturm-Liouville problems with integral conditions, little has been done.

In this work we concern ourselves with the reconstruction of the SturmLiouville equations with nonlocal boundary conditions from nodal data. First, qualitative behaviour of eigenvalues subject to nonlocal integral boundary conditions and oscillation of the corresponding eigenfunctions are described. Second, the asymptotic expression of nodal of eigenfunctions is given. Finally, we prove the corresponding uniqueness theorem and provide a constructive procedure for the solution.

\section{Main Results}

Let us consider the following boundary-value problem for the Sturm-Liouville operator on segment $[0, \pi]$ :

$$
-y^{\prime \prime}+q(x) y=\lambda y, \lambda=s^{2}
$$

with one classical boundary condition

$$
y^{\prime}(0)-h y(0)=0, \quad h \in \mathbf{R} \cup\{\infty\}
$$

and another nonlocal integral boundary condition

$$
y(\pi)=\int_{0}^{\pi} y(x) \mu(x) d x
$$

where $q(x)$ and $\mu(x)$ are real-valued, twice differentiable functions on segment $[0, \pi]$. In particular, if $h=\infty$, the classical boundary condition is

$$
y(0)=0 .
$$

The differential operation (2.1) together with boundary conditions (2.2) and (2.3) define a linear (non-self-adjoint) operator in the Hilbert space $L^{2}[0, \pi]$, and 
the problem (2.1)-(2.3) is an eigenvalue problem for this operator. From the results of [4] it follows that the spectrum of the operator is discrete and consists of single eigenvalues. Let $\lambda_{0}, \lambda_{1}, \cdots, \lambda_{n}, \cdots$ be the eigenvalues of the problem (2.1) $-(2.3)$, ordered by increasing modulus.

Let $\phi(x, \lambda)$ be the solution of (2.1) satisfying the following initial conditions

$$
\phi(0, \lambda)=1, \quad \phi^{\prime}(0, \lambda)=h .
$$

Then the spectra of the boundary-value problem (2.1)-(2.3) are the zerosequences $\left\{\lambda_{n}\right\}_{n=0}^{\infty}$ of the entire function

$$
\phi(\pi, \lambda)-\int_{0}^{\pi} \phi(x, \lambda) \mu(x) d x=0 .
$$

Let $\phi\left(x, \lambda_{n}\right)$ be the eigenfunction corresponding to the eigenvalue $\lambda_{n}$ of the Sturm-Liouville operator (2.1)-(2.3). Using an analog of the Sturm oscillation theorem, for sufficiently large $n$ we get $\phi\left(x, \lambda_{n}\right)$ has exactly $n-1$ nodal points in $(0, \pi)(h=\infty)$, and exactly $n$ nodal points in $(0, \pi)(h \neq \infty)$. Suppose $x_{n}^{j}$ are the nodal points of the eigenfunction $\phi\left(x, \lambda_{n}\right)$. In other words, $\phi\left(x_{n}^{j}, \lambda_{n}\right)=0$. Let $I_{n}^{j}=\left(x_{n}^{j}, x_{n}^{j+1}\right)$, and the nodal length $l_{n}^{j}$ by $l_{n}^{j}=x_{n}^{j+1}-x_{n}^{j}$. We also define the function $j_{n}(x)$ to be the largest index $j$ such that $0 \leq x_{n}^{j} \leq x$.

Denote $X:=\left\{x_{n}^{j}\right\}$. $X$ is called the set of nodal points of the Sturm-Liouville operator (2.1)-(2.3). We consider the following inverse problem.

Problem: Given nodal points set $X$ or its subset $X_{0}$ which is dense in $(0, \pi)$, find the boundary condition parameter $h$ and the potential $q(x)$.

The main theorems are the following.

Theorem 1. Fix $x \in[0, \pi]$. Let $\left\{x_{n}^{j}\right\} \subset X$ be chosen such that $\lim _{n \rightarrow \infty} x_{n}^{j}=x$. Then the following finite limits exist and the corresponding equalities hold.

(a) In the case $h \neq \infty$.

$$
\begin{aligned}
& \lim _{n \rightarrow \infty}\left[n^{2} x_{n}^{j}-n\left(j-\frac{1}{2}\right) \pi-\frac{1}{2}\left(j-\frac{1}{2}\right) \pi\right]:=f(x), \\
& f(x)=h-\frac{h x}{\pi}-\frac{x}{2 \pi} \int_{0}^{\pi} q(t) d t+\frac{\mu(\pi) x}{\pi}+\frac{x}{4}+\frac{1}{2} \int_{0}^{x} q(t) d t .
\end{aligned}
$$

(b) In the case $h=\infty$ and $n$ is chosen even number series.

$$
\begin{aligned}
& \lim _{n \rightarrow \infty} n^{2}\left(x_{n}^{j}-\frac{j \pi}{n}\right):=g(x), \\
& g(x)=\frac{1}{2} \int_{0}^{x} q(t) d t-\frac{x}{\pi}\left[\mu(0)-\mu(\pi)+\frac{1}{2} \int_{0}^{\pi} q(t) d t\right] .
\end{aligned}
$$

Let us now formulate a uniqueness theorem and provide a constructive procedure for the solution of the inverse nodal problem.

Theorem 2. Let $X_{0} \subset X$ be a subset of nodal points which is dense in $(0, \pi)$. Then, the specification of $X_{0}$ uniquely determines the potential

$$
q(x)-\frac{1}{\pi} \int_{0}^{\pi} q(x) d x \quad \text { in }(0, \pi)
$$


and the coefficient $h$ of the boundary conditions. The number $h$ and the potential $q(x)-\frac{1}{\pi} \int_{0}^{\pi} q(x) d x$ can be constructed via the following algorithm:

(1) for each $x \in[0, \pi]$ choose a sequence $\left\{x_{n}^{j}\right\} \subset X_{0}$ such that $x_{n}^{j} \rightarrow x$ as $n \rightarrow \infty$ (note in the case $h=\infty$ we choose $n$ an even number series);

(2) find the functions $f(x)$ and $g(x)$ via (2.4)-(2.7)) and in turn, calculate

(a) In the case $h \neq \infty$ :

$$
\begin{aligned}
& \mu(\pi)=f(\pi)-\frac{\pi}{4}, \quad h=f(0) \\
& q(x)-\frac{1}{\pi} \int_{0}^{\pi} q(t) d t=2 f^{\prime}(x)+\frac{2 h}{\pi}-\frac{2 \mu(\pi)}{\pi}-\frac{1}{2}
\end{aligned}
$$

(b) In the case $h=\infty$ :

$$
\mu(\pi)-\mu(0)=g(\pi), \quad q(x)-\frac{Q(\pi)}{\pi}=2 g^{\prime}(x)+\frac{2}{\pi}[\mu(0)-\mu(\pi)] .
$$

Using only the nodal data, we can reconstruct the potential. Our reconstruction formulae are direct and automatically it implies the uniqueness of this inverse problem $(2.1)-(2.3)$.

\section{Proofs}

We now proceed with the proof of theorems in the case $h \neq \infty$. The other case is treated similarly. First qualitative behaviour of eigenvalues for SturmLiouville operator (2.1)-(2.3) is given.

Lemma 1. For sufficiently large $n$, qualitative behaviour of eigenvalues $\lambda_{n}=$ $s_{n}^{2}$ for Sturm-Liouville operator (2.1)-(2.3) is as follows

(a) In the case $h \neq \infty$ :

$$
s_{n}=n-\frac{1}{2}+\frac{1}{n \pi}\left[h-\mu(\pi)+\frac{1}{2} \int_{0}^{\pi} q(t) d t\right]+O\left(\frac{1}{n^{2}}\right) .
$$

(b) In the case $h=\infty$ :

$$
s_{n}=n+\frac{1}{\pi n}\left[(-1)^{n} \mu(0)-\mu(\pi)+\frac{1}{2} \int_{0}^{\pi} q(t) d t\right]+O\left(\frac{1}{n^{2}}\right) .
$$

Proof. The solution $\phi\left(x, \lambda_{n}\right)$ has the following asymptotic formula for $n \rightarrow \infty$ uniformly in $x[16]$ :

$$
\phi(x, \lambda)=\cos s x+\frac{h}{s} \sin s x+\frac{\sin s x}{2 s} \int_{0}^{x} q(t) d t+O\left(\frac{e^{\tau \pi}}{s^{2}}\right), \quad \tau=|\operatorname{Im} s| .
$$

Note that

$$
\begin{aligned}
\int_{0}^{\pi} y(x) \mu(x) d x & =\int_{0}^{\pi}\left[\cos s x+\frac{h}{s} \sin s x+\frac{\sin s x}{2 s} \int_{0}^{x} q(t) d t+O\left(\frac{e^{\tau \pi}}{s^{2}}\right)\right] \mu(x) d x \\
& =\frac{\sin s \pi}{s} \mu(\pi)+O\left(\frac{e^{\tau \pi}}{s^{2}}\right) .
\end{aligned}
$$


Substituting the expression $\phi(x, \lambda)$ into $(2.3)$, we obtain

$$
\begin{aligned}
& \cos s \pi+\frac{h}{s} \sin s \pi+\frac{\sin s \pi}{2 s} \int_{0}^{\pi} q(t) d t-\frac{\sin s \pi}{s} \mu(\pi)+O\left(\frac{e^{\tau \pi}}{s^{2}}\right)=0 \\
& -\tan (s \pi+\pi / 2)=\cot s \pi=-\frac{h}{s}-\frac{1}{2 s} \int_{0}^{\pi} q(t) d t+\frac{1}{s} \mu(\pi)+O\left(\frac{1}{s^{2}}\right), \\
& s_{n}=n-\frac{1}{2}+\frac{1}{n \pi}\left[h-\mu(\pi)+\frac{1}{2} \int_{0}^{\pi} q(t) d t\right]+O\left(\frac{1}{n^{2}}\right) .
\end{aligned}
$$

This proves the lemma.

Next we prove that an analog of the classical Sturm's oscillation theorem for the Sturm-Liouville operator (2.1)-(2.3).

Lemma 2. For sufficiently large $n$, the eigenfunction $\phi\left(x, \lambda_{n}\right)$ of the SturmLiouville operator (2.1)-(2.3) has exactly $n-1$ nodal points in $(0, \pi)(h=\infty)$, and exactly $n$ nodal points in $(0, \pi)(h \neq \infty)$. Moreover, in the case $h \neq \infty$ :

$$
\begin{aligned}
x_{n}^{j}= & \frac{\left(j-\frac{1}{2}\right) \pi}{n}+\frac{h}{n^{2}}+\frac{\left(j-\frac{1}{2}\right) \pi}{2 n^{2}}-\frac{\left(j-\frac{1}{2}\right) h}{n^{3}}-\frac{\left(j-\frac{1}{2}\right)}{2 n^{3}} \int_{0}^{\pi} q(t) d t \\
& +\frac{\left(j-\frac{1}{2}\right) \mu(\pi)}{n^{3}}+\frac{\left(j-\frac{1}{2}\right) \pi}{4 n^{3}}+\frac{1}{2 n^{2}} \int_{0}^{x_{n}^{j}} q(t) d t+O\left(\frac{1}{n^{3}}\right)
\end{aligned}
$$

and in the case $h=\infty$ :

$$
x_{n}^{j}=\frac{j \pi}{n}+\frac{1}{2 n^{2}} \int_{0}^{x_{n}^{j}} q(t) d t-\frac{j}{n^{3}}\left[(-1)^{n} \mu(0)-\mu(\pi)+\frac{1}{2} \int_{0}^{\pi} q(t) d t\right]+O\left(\frac{1}{n^{3}}\right),
$$

uniformly with respect to $j \in \mathbf{Z}^{+}$.

Proof. In the case $h \neq \infty$, the eigenfunction $\phi\left(x, \lambda_{n}\right)$ has the following asymptotic formula for $n \rightarrow \infty$ uniformly in $x[16]$ :

$$
\phi\left(x, \lambda_{n}\right)=\cos \left(s_{n} x\right)+\frac{h \sin \left(s_{n} x\right)}{s_{n}}+\frac{\sin \left(s_{n} x\right)}{2 s_{n}} \int_{0}^{x} q(t) d t+O\left(\frac{e^{\tau \pi}}{s_{n}^{2}}\right) .
$$

Then, for the nodal point $x_{n}^{j}$ of the eigenfunction $\phi\left(x, \lambda_{n}\right)$, from

$$
0=\phi\left(x_{n}^{j}, \lambda\right)=\cos \left(s_{n} x\right)+\frac{h \sin \left(s_{n} x\right)}{s_{n}}+\frac{\sin \left(s_{n} x\right)}{2 s_{n}} \int_{0}^{x} q(t) d t+O\left(\frac{e^{\tau \pi}}{s_{n}^{2}}\right)
$$

we obtain

$$
\cot \left(s_{n} x_{n}^{j}\right)=-\frac{h}{s_{n}}-\frac{1}{2 s_{n}} \int_{0}^{x_{n}^{j}} q(t) d t+O\left(\frac{1}{n^{2}}\right) .
$$

Using Taylor's expansions, we obtain the following asymptotic formula for nodal points as $n \rightarrow \infty$ uniformly in $j \in \mathbf{Z}^{+}$:

$$
s_{n} x_{n}^{j}=\left(j-\frac{1}{2}\right) \pi+\frac{h}{s_{n}}+\frac{1}{2 s_{n}} \int_{0}^{x_{n}^{j}} q(t) d t+O\left(\frac{1}{n^{2}}\right),
$$


which implies

$$
x_{n}^{j}=\frac{\left(j-\frac{1}{2}\right) \pi}{s_{n}}+\frac{h}{s_{n}^{2}}+\frac{1}{2 s_{n}^{2}} \int_{0}^{x_{n}^{j}} q(t) d t+O\left(\frac{1}{n^{3}}\right) .
$$

Moreover, using the asymptotic formulae

$$
s_{n}^{-1}=\frac{1}{n}+\frac{1}{2 n^{2}}-\frac{h}{n^{3} \pi}-\frac{\int_{0}^{\pi} q(t) d t}{2 n^{3} \pi}+\frac{\mu(\pi)}{n^{3} \pi}+\frac{1}{4 n^{3}}+O\left(\frac{1}{n^{4}}\right), s_{n}^{-2}=\frac{1}{n^{2}}+O\left(\frac{1}{n^{3}}\right),
$$

thus, we have

$$
\begin{aligned}
x_{n}^{j}= & \frac{\left(j-\frac{1}{2}\right) \pi}{n}+\frac{h}{n^{2}}+\frac{\left(j-\frac{1}{2}\right) \pi}{2 n^{2}}-\frac{\left(j-\frac{1}{2}\right) h}{n^{3}}-\frac{\left(j-\frac{1}{2}\right)}{2 n^{3}} \int_{0}^{\pi} q(t) d t+\frac{\left(j-\frac{1}{2}\right) \mu(\pi)}{n^{3}} \\
& +\frac{\left(j-\frac{1}{2}\right) \pi}{4 n^{3}}+\frac{1}{2 n^{2}} \int_{0}^{x_{n}^{j}} q(t) d t+O\left(\frac{1}{n^{3}}\right) .
\end{aligned}
$$

The equality (3.1) holds. It gives

$$
x_{n}^{j+1}-x_{n}^{j}:=l_{n}^{j}=\frac{\pi}{n}+O\left(\frac{1}{n^{2}}\right), n \rightarrow \infty
$$

uniformly with respect to $j$. In the case $h \neq \infty$, for $j=0,1, \cdots, n+1$, the formula (3.1) gives

$$
\begin{aligned}
& x_{n}^{0}=\frac{-\frac{1}{2} \pi}{n}+O\left(\frac{1}{n^{2}}\right), \quad x_{n}^{1}=\frac{\frac{1}{2} \pi}{n}+O\left(\frac{1}{n^{2}}\right), x_{n}^{2}=\frac{\frac{3}{2} \pi}{n}+O\left(\frac{1}{n^{2}}\right), \\
& \ldots, x_{n}^{n}=\frac{\left(n-\frac{1}{2}\right) \pi}{n}+O\left(\frac{1}{n}\right), \quad x_{n}^{n+1}=\frac{\left(n+\frac{1}{2}\right) \pi}{n}+O\left(\frac{1}{n}\right) .
\end{aligned}
$$

Thus, according to the order of $x_{n}^{j}$, for large $n$, the eigenfunction $\phi\left(x, \lambda_{n}\right)$ of the Sturm-Liouville operator has exactly $n$ nodes in the interval $(0, \pi)$, i.e., $x_{n}^{j}, j=\overline{1, n}$.

In the above results, the order estimate is independent of $j$. As a result, we get that $l_{n}^{j}=\pi / n+o(1 / n)$.

Corollary 1. From Lemma 2 it follows that the sets $X=\left\{x_{n}^{j}\right\}$ are dense in $[0, \pi]$.

Now we can give the proofs of the theorems.

Proof. [Proof of Theorem 1] Using the asymptotic expansions (3.1) and (3.3) for nodal points and the fact that $\lim _{n \rightarrow \infty} x_{n}^{j}=x$, we get

$$
\begin{aligned}
& \lim _{n \rightarrow \infty}\left[n^{2} x_{n}^{j}-n\left(j-\frac{1}{2}\right) \pi-\frac{1}{2}\left(j-\frac{1}{2}\right) \pi\right]=\lim _{n \rightarrow \infty}\left[h-\frac{\left(j-\frac{1}{2}\right) h}{n}\right. \\
& \left.\quad-\frac{\left(j-\frac{1}{2}\right)}{2 n} \int_{0}^{\pi} q(t) d t+\frac{\left(j-\frac{1}{2}\right) \mu(\pi)}{n}+\frac{\left(j-\frac{1}{2}\right) \pi}{4 n}+\frac{1}{2} \int_{0}^{x_{n}^{j}} q(t) d t+O\left(\frac{1}{n}\right)\right]
\end{aligned}
$$




$$
=h-\frac{h x}{\pi}-\frac{x}{2 \pi} \int_{0}^{\pi} q(t) d t+\frac{\mu(\pi) x}{\pi}+\frac{x}{4}+\frac{1}{2} \int_{0}^{x} q(t) d t \triangleq f(x) .
$$

This proves the theorem.

Proof. [Proof of Theorem 2] For a given nodal subset $X_{0}$, by Theorem 1 we can build up the reconstruction formulae. Formulae (2.8) and (2.9) can be derived from (2.4) and (2.7) stepwise. We obtain the following procedure.

For the case $h \neq \infty$ :

- Step 1. Take $x=\pi$, it follows $\mu(\pi)=f(\pi)-\frac{\pi}{4}$.

- Step 2. Take $x=0$, then it yields $h=f(0)$.

- Step 3. By taking derivatives we obtain

$$
q(x)-\frac{1}{\pi} \int_{0}^{\pi} q(t) d t=2 f^{\prime}(x)+\frac{2 h}{\pi}-\frac{2 \mu(\pi)}{\pi}-\frac{1}{2} .
$$

For the case $h=\infty$ :

- Step 1. Take $x=\pi$, it follows $\mu(\pi)-\mu(0)=g(\pi)$.

- Step 2. By taking derivatives we obtain

$$
q(x)-\frac{1}{\pi} \int_{0}^{\pi} q(t) d t=2 g^{\prime}(x)+\frac{2}{\pi}[\mu(0)-\mu(\pi)] .
$$

Thus these formulae are constructed. Since each nodal data determines one set of reconstruction formulae which only depend on nodal data, the uniqueness holds obviously.

\section{Conclusions}

In fact, using the methods in this paper we can obtain a extended conclusion.

Consider the following boundary-value problem for the Sturm-Liouville operator on segment $[0, \pi]$ :

$$
-y^{\prime \prime}+q(x) y=\lambda y
$$

with one classical boundary condition

$$
y^{\prime}(0)-h y(0)=0, \quad h \in \mathbf{R} \cup\{\infty\}
$$

and another nonlocal integral boundary condition

$$
y^{\prime}(\pi)+H y(\pi)=\int_{0}^{\pi} y(x) \mu(x) d x, \quad H \in \mathbf{R}
$$

where $q(x)$ and $\mu(x)$ are real-valued, twice differentiable functions on segment $[0, \pi]$. In particular, if $h=\infty$, the classical boundary condition is

$$
y(0)=0 .
$$

A uniqueness theorem of the inverse nodal problem (4.1), (4.2) and (4.3) is the following; its proof is similar to that of Theorem 2 . 
Theorem 3. Let $X_{0}$ be a subset of nodal points which is dense in $(0, \pi)$ for the inverse nodal problem (4.1), (4.2) and (4.3). Then, the specification of $X_{0}$ uniquely determines the potential $q(x)-\frac{1}{\pi} \int_{0}^{\pi} q(x) d x$ in $(0, \pi)$, and the coefficients $h$ and $H$ of the boundary conditions.

\section{Acknowledgments}

The author acknowledges helpful comments and suggestions from the referees.

\section{References}

[1] S. Albeverio, R. O. Hryniv and L. P. Nizhnik. Inverse spectral problems for non-local Sturm-Liouville operators. Inverse Problems, 23:523-535, 2007. Doi:10.1088/0266-5611/23/2/005.

[2] A. V. Bitsadze and A. A. Samarskii. Some elementary generalizations of linear elliptic boundary value problems. Dokl. Akad. Nauk SSSR, 185:739-740, 1969.

[3] P. J. Browne and B. D. Sleeman. Inverse nodal problem for Sturm-Liouville equation with eigenparameter dependent boundary conditions. Inverse Problems, 12:377-381, 1996. Doi:10.1088/0266-5611/12/4/002.

[4] V. Bryuns. Generalized boundary-value problem for an ordinary linear differential operator. Dokl. Akad. Nauk SSSR, 198(6):1255-1258, 1971.

[5] S. A. Buterin and C. T. Shieh. Inverse nodal problem for differential pencils. Applied Mathematics Letters, 22:1240-1247, 2009. Doi:10.1016/j.aml.2009.01.037.

[6] B. Chanane. Computing the eigenvalues of a class of nonlocal Sturm-Liouville problems. Mathematical and Computer Modelling, 50:225-232, 2009.

[7] Y. T. Chen, Y. H. Cheng, C. K. Law and J. Tsay. $L^{1}$ convergence of the reconstruction formula for the potential function. Proc. Amer. Math. Soc., 130:23192324, 2002. Doi:10.1090/S0002-9939-02-06297-4.

[8] Y. H. Cheng, C. K. Law and J. Tsay. Remarks on a new inverse nodal problem. J. Math. Anal. Appl., 248:145-155, 2000. Doi:10.1006/jmaa.2000.6878.

[9] R. Čiupaila, Ž. Jesevičiūtè and M. Sapagovas. Eigenvalue problem for ordinary differential operator subject to integer condition. Nonlinear Analysis: Modelling and Control, 9(2):109-116, 2004.

[10] S. Currie and B. A. Watson. Inverse nodal problems for Sturm-Liouville equations on graphs. Inverse Problems, 23:2029-2040, 2007.

Doi:10.1088/0266-5611/23/5/013.

[11] L. Greenberg and M. Marletta. Numerical solution of non-self-adjoint SturmLiouville problems and related systems. SIAM Journal on Numerical Analysis, 38(6):1800-1845, 2001. Doi:10.1137/S0036142999358743.

[12] O. H. Hald and J. R. McLaughlin. Solutions of inverse nodal problems. Inverse Problems, 5:307-347, 1989.

[13] G. Infante. Eigenvalues of some non-local boundary-value problems. Proceedings of the Edinburgh Mathematical Society, 46:75-86, 2003. Doi:10.1017/S0013091501001079.

[14] N. I. Ionkin and E. A. Valikova. On eigenvalues and eigenfunctions of a nonclassical boundary value problem. Math. Modelling, Russia, 1(8):53-63, 1996. (in Russian) 
[15] H. Koyunbakan. A new inverse problem for the diffusion operator. Appl. Math. Lett., 19:995-999, 2006. Doi:10.1016/j.aml.2005.09.014.

[16] C. K. Law, C. L. Shen and C. F. Yang. The inverse nodal problem on the smoothness of the potential function. Inverse Problems, 15:253-263, 1999. Errata, 17, (2001) 361-364.

[17] C. K. Law and J. Tsay. On the well-posedness of the inverse nodal problem. Inverse Problems, 17:1493-1512, 2001. Doi:10.1088/0266-5611/17/5/317.

[18] C. K. Law and C. F. Yang. Reconstructing the potential function and its derivatives using nodal data. Inverse Problems, 14(2):299-312, 1998. Doi:10.1088/0266-5611/14/2/006.

[19] B. M. Levitan. Inverse Sturm-Liouville Problems. Utrecht: VNU Science Press, 1987.

[20] B. M. Levitan and I. S. Sargsjan. Sturm-Liouville and Dirac Operators. Nauka, Moscow (in Russian), 1988. English transl., Kluwer, Dordrecht, 1991.

[21] V. A. Marchenko. Sturm-Liouville Operators and Their Applications. Naukova Dumka, Kiev, 1977. English transl.: Birkhäser, 1986.

[22] J. R. McLaughlin. Inverse spectral theory using nodal points as data-a uniqueness result. J. Differential Equations, 73:354-362, 1988.

Doi:10.1016/0022-0396(88)90111-8.

[23] J. Pöschel and E. Trubowitz. Inverse Spectral Theory. Academic Press, Orlando, 1987.

[24] S. Pečiulytė, O. Štikonienė and A. Štikonas. Sturm-Liouville problem for stationary differential operator with nonlocal integral boundary conditions. Math. Model. Anal., 10(4):377-392, 2005.

[25] M. P. Sapagovas. The eigenvalues of some problem with a nonlocal condition. Diff. Equations, 7(38):1020-1026, 2002. Doi:10.1023/A:1021115915575. (in Russian)

[26] M. P. Sapagovas and A. D. Śtikonas. On the structure of the spectrum of a differential operator with a nonlocal condition. Diff. Equations, 41(7):10101018, 2005. Doi:10.1007/s10625-005-0242-y.

[27] C. L. Shen. On the nodal sets of the eigenfunctions of the string equations. SIAM J. Math. Anal., 19:1419-1424, 1988. Doi:10.1137/0519104.

[28] C. L. Shen and C. T. Shieh. An inverse nodal problem for vectorial SturmLiouville equation. Inverse Problems, 16:349-356, 2000.

Doi:10.1088/0266-5611/16/2/306.

[29] C. T. Shieh and V. A. Yurko. Inverse nodal and inverse spectral problems for discontinuous boundary value problems. J. Math. Anal. Appl., 347:266-272, 2008. Doi:10.1016/j.jmaa.2008.05.097.

[30] A. Štikonas and O. Štikonienè. Characteristic functions for Sturm-Liouville probleams with nonlocal boundary conditions. Math. Model. Anal., 44(2):229-246, 2009.

[31] X. F. Yang. A solution of the inverse nodal problem. Inverse Problems, 13:203213, 1997.

[32] V. A. Yurko. Integral transforms connected with discontinuous boundary value problems. Integral Transforms Spec. Funct., 10(2):141-164, 2000. 
[33] V. A. Yurko. Inverse Spectral Problems for Differential Operators and Their Applications. Gordon and Breach, Amsterdam, 2000.

[34] V. A. Yurko. Inverse nodal problems for Sturm-Liouville operators on star-type graphs. J. Inv. Ill-Posed Problems, 16:715-722, 2008. 